\title{
Entropy Driven Bit Coding For Image Compression In Medical Application
}

\author{
S. Jagadeesh, E. Nagabhooshanam \\ Research Scholar, Dept. of ECE, SSJ Engineering College/ JNTUH, Hyderabad, India. \\ Professor, Dept. of ECE, Sridevi Women's Engineering College/ JNTUH, Hyderabad, India.
}

\begin{abstract}
This paper present a new bit redundancy coding for image compression in binary bit planar coding. In the compression model, images are coded into binary level to stream over a communication medium or to store the processed data in a remote location. In this processing, the representative coefficients are coded in binary level and to minimize the resource overhead these bits are compressed using binary compression logic. Among different coding logic, Huffman coding is the standard coding approach, standardized by JPEG and JPEG-2K image compression committee. The coding schemes computes a bit pattern occurrence probability and derive a allocating code word for a pattern to be compressed. The advantage of utilizing redundancy coding result in higher compression. However, the over-coding issue for lower pattern probability gives a inverse compression affect in image compression. In this paper, this issue is addressed an a new hybrid image coding approach developing a mixed model approach of variable entropy coding and fixed pattern allotment is proposed. The proposed hybrid approach termed "Selective Hybrid Coding" is used for the compression of medical samples and compared for performance evaluation to the conventional image compression model.
\end{abstract}

Index Terms: Image compression, medical image, entropy coding, Huffman coding, hybrid coding

\section{Introduction}

Image compression is been a area of research from long time.Standards for the efficient representation and interchange of digital image are essential. To date some of the most successful still image compression standards have resulted from the ongoing work of the Joint photographic Experts Group (JPEG). Basically a JPEG system consists of a Transformation unit followed by Quantizer and Encoder unit under image encoding. Under the Decoding side the Data is retrieved using the reverse operation to the Encoding operation. During the progressive transmission it is a basic approach of compressing the data before transmission so as to improve the rate of transmission with minimum bandwidth requirement. For the compression of image data various compression approaches were proposed in past. These coding approaches are lossy or lossless compression approach as per the developed methods. In these approaches more dominantly used approaches are Huffman coding and arithmetic coding approach. As Huffman coding are lower in computational complexity as compared to arithmetic coding mostly Huffman coding are seen in JPEG coding architecture. Huffman coding is entropy based coding technique where the data bits are compressed based on the frequency of occurrence of the unique words. It is often observed that this coding approach allocated higher code bit length than the original code word for lower frequency data bits. This result in decompression and result in higher bit counts. Additionally the decoding states required to decompress the data increases resulting in slower operational speed. To achieve a higher compression and faster decoding time in this work an hybrid coding approach of variable and fixed length coding scheme is to be developed for the improvement of speed and compression ratio for digital image compression. For the compression of medical images various image compression approaches were proposed in past. The conventional image compression approaches such as JPEG [1], JPEG-2000 [2], SPHIT[3], EBCOT[4], Lifting scheme[5] etc. were proposed earlier. These approaches are majorly categorized under lossy or lossless compression schemes. In lossy compression [6] the information are not accurately retrieved at the receiver side resulting in low PSNR. These methods are basically suitable for faster Encodingapproach. In various scenarios where degradation of image is not tolerable, lossless compression schemes were proposed. Lossless compression scheme [7] is a method that allows the exact accurate original data to be reconstructed from the compressed data. A scheme such as wavelet-based compression with adaptive prediction [8] is a lossless approach of image compression. This scheme is mainly used to achieve higher compression ratio. For obtaining a lossless compression in [9] a lifting scheme is suggested based on adaptive threshold. In medical area one cannot afford information loss due to compact coding, because lossy compression may destroy some of the information required during processing, or add artifacts that lead to erroneous interpretations [10]. Lossless compression has been widely preferred by medical professionals, because it facilitates accurate diagnosis. The standard wavelet compression techniques [11], even if lossless in principle, do not reconstruct exactly the original image because of the rounding of the floating-point wavelet coefficients to integers. The use of the lifting scheme allows generating truly loss-less integer-to-integer wavelet transforms. In recent years, new 
reversible integer wavelet transforms can be developed using lifting scheme [12], have grown in popularity for lossless image compression [13]. These compression systems comprising a wavelet transform followed by Shapiro's EZW (embedded zero trees of wavelet coefficient) algorithm [14], have proven to be a successful method in the field of image compression [43]. In this paper zero tree coding scheme together with new modified runlength coding is used to encode the subband coefficients [15]. Modified runlength coding (MRLE) increases the compression ratios of the proposed approach and it also increase the security level [16]. Lossless image compression [17] coder, such as context based predictive coding (CALIC) and universal context modeling based coding in [18]. The lack of a progressive Encoding capability makes them unsuitable for interactive applications like telematics over large networks. Embedded coding [19] permits the progressive Encoding of the compressed data, starting with an economical initial Encoding of a rough image followed by gradual Encoding of the refinement details, needed for the final reconstruction. Embedded progressive coding becomes quite important in terms of compressing medical images, because an image may be stored without any loss.

\section{Compression Model}

The JPEG-2000 image compression architecture is the fundamental architecture consisting an encoder and decoder unit. The function of the encoder is, when an input is given, it creates a set of symbols from the given input data and then the data is transmitted through a channel and then feed to decoder where we can reconstruct the image given as input. There is a possibility that the reconstructed output image can the replica of the input image or the reconstructed image can have distortion is due to channel interference. Figure. 1 shows convention block diagram of a compression system.

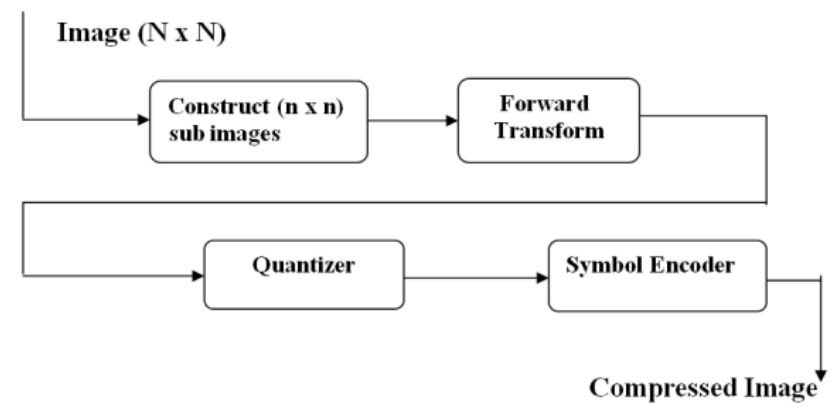

Figure 1: Block Diagram of an Image compression system.

In the process of encoding the image is preprocessed for filtration and output is then processed using DWT. To perform the forward DWT the JPEG2000 system uses a one-dimensional (1-D) subband decomposition of a 1-D set of samples into low-pass and high-pass samples. Quantization refers to the process of approximating the continuous set of values in the image data with a finite (preferably small) set of values. After the data has been quantized into a finite set of values, it can be encoded using an Entropy Coder to give additional compression. By entropy, it means the amount of information present in the data, and an entropy coder encodes the given set of symbols with the minimum number of bits required to represent them using Huffman coding. The Huffman decoder block carries out decoding reading the unique code bits passed in place of the data bit. The dequantizer unit dequantizes the decoded data bits. Inverse transformation is the process of retrieving back the image data from the obtained image values. The image data transformed and decomposed under encoding side is rearranged from higher level decomposition to lower level with the highest decomposed level been arranged at the top. In the compression process image are coded using transformation domain where the input image is transformed using discrete cosine transform (DCT) and discrete wavelet transform (DWT). In the transformed domain the coding wavelet based coding is observed to be more optimal in comparison to conventional DCT technique. The multi resolution property gives the approach a higher advantage over DCT coding. Though the MRA approach has the advantage of higher resolution processing, higher resource constraints are observed in such coding. The band redundancy is the primal issue which build a coding overhead in wavelet domain coding.

\section{Correlative Band Selective Logic}

The compression in wavelet domain has the advantage of MRA; however the coding overhead is large. To minimize the coding overhead a correlative band selection logic is proposed in [20]. The probability of redundancy among different bands increases. This redundancy of information increases the processing overhead, and intern makes the system slower. Hence it is required to have an adaptive band selection process for extracting the actual informative band from the processed bands. With reference to band selection process in 
[20] the process of adaptive band selection is developed for multi wavelet coefficients. Considering the analysis and synthesis filter of the transformation is proposed. In this process the analysis bank decomposes the image I into $\mathrm{K}$ subbands, each produced by a branch $\mathrm{H}_{\mathrm{z}}(\mathrm{k})$ of the analysis bank. After decimation and expansion by a factor $\mathrm{N}$, the full band signal is reconstructed from the subbands in the synthesis bank by filtering with filters $\mathrm{G}_{\mathrm{k}}(\mathrm{z})$ followed by summation. The analysis filters $\mathrm{H}_{\mathrm{k}}(\mathrm{n})$ are derived from the real value of a lowpass FIR filter $\mathrm{p}[\mathrm{n}]$ of even length $\mathrm{L}_{\mathrm{p}}$. For the estimation of signal using such filtration cost optimization approached is used. This approach sorts out a subset of the subband filters contributing to convergence performance and utilizes those in updating the adaptive filter weight. This approach dynamically selects the subband filters so as to fulfill the largest decrease of the successive mean square deviations (MSDs) at every iteration. This approach reduces the computational complexity with critical sampling while maintaining its selection performance. To optimize the significant coefficient selection a spectral energy based coefficient selection is proposed. Spectral energy based compression is a more effective approach in compression. However, in this approach Wavelet coefficients were processed. Over the normalized coefficients rather to linearly applying compression, coefficients with lower energy density are selected. This integral approach results in optimal selection of significant coefficients, which results in best coding performance. In this approach a power spectral density (PSD) is computed for low variation regions.For the selection of the selective coefficient of the selected spectrum, a coefficient selection algorithm is proposed. The developed approach is termed as "Spectral selective coding" (SSC). The process of selective coding is as outlined; For the selected Normalized band ' $\mathrm{BN}_{\mathrm{i}}$ ' obtained from the decomposition approach, a decision of coefficient selection is made based on spectral magnitude. This approach of coefficient selection, results in selection of coefficients, at lower frequency level without effecting higher resolutions coefficients. To compute the spectral magnitude of the band coefficient a power spectral densities (PSD) is computed. PSD is defined as a density operator which defines the variation of power over different content frequencies, in a given signal $\mathrm{x}(\mathrm{t})$. Taking the selected coefficients, the compression process is made. For the compression of coefficients then a SPHIT coding is used. Wherein the encoded data bits are then coded using entropy encoder using Huffman coding. The property of entropy encoding is used.

\section{Entropy Driven Bit Coding}

Two statistical methods of data compression namely Huffman and Hybrid Huffman coding are used here for the compression/ Decompression of test set. Further analyses on the two methods are carried out with reference to the speed of encoding and decoding, the memory used for the storage of these test sets. A total of 12 × 32 (384 test bits) are considered for testing with 4-bit Blocks taken for the operation. The system consists of an encoder block and a decoder block for the compression and decompression of the test set considered. Given the test set for a core, a statistical code for compressing the test set must be selected. There is a tradeoff in selecting the code between the amount of compression that is achieved and the complexity of the decoder. Moreover, if the clock frequency of the tester is $\mathrm{f}_{\mathrm{T}}$ and the clock frequency of the scan chain is $\mathrm{f}_{\text {sys }}$ (system clock frequency) then the ratio of the system clock frequency and the tester clock frequency $\mathrm{f}_{\text {sys }}=\mathrm{f}_{\mathrm{T}}$ limits the minimum size of a codeword. The compression/decompression scheme described in this paper is based on hybrid Huffman coding. In statistical coding, variable length code words are used to represent fixed-length blocks of bits in a data set. For example, if a data set is divided into four-bit blocks, then there are 24 or 16 unique four-bit blocks. Each of the 16 possible four-bit blocks can be represented by a binary codeword. The size of each codeword is variable (it need not be four bits). The idea is to make the code words that occur most frequently have a smaller number of bits, and those that occur least frequently to have a larger number of bits. This minimizes the average length of a codeword. The goal is to obtain a coded representation of the original data set that has the smallest number of bits.Suppose the scan vectors are divided into four-bit blocks, and each four-bit block is replaced by a variable length codeword. The compressed test data stored on the tester consists of the variable length code words. These code words are shifted into the decoder as a continuous stream of bits. If the code words are prefix-free, than the decoder can easily recognize when it has received a complete codeword. When the decoder has received a complete codeword, it loads the corresponding four-bit block in parallel into the serializer. The contents of the serializer are then shifted into the scan chain. If the scan chain is clocked at twice the clock rate that the tester operates at, then after two tester clock periods the entire contents of the serializer will be shifted into the scan chain. During the two tester clock periods that the serializer is in operation, the decoder can be receiving the next codeword. The key to making the scheme work is careful selection of the statistical code that is used for compressing the test set. There are two important issues that must be considered in selecting the code: one is that the decoder must be small in order to keep the area overhead down, and the other is that the decoder must not output the decompressed bits into the serializer faster than they can be shifted out into the scan chain. While a Huffman code gives the optimum compression for a test set divided into a particular fixed-length block size, it generally requires a very large decoder. A Huffman code for a fixed-length block size of $b$ bits requires a finite state machine (FSM) decoder with $2_{b-1}$ states. Thus, the size of the decoder for a Huffman code grows exponentially as the block size is increased. 
Given the test set for a core, a statistical code for compressing the test set must be selected. There is a tradeoff in selecting the code between the amount of compression that is achieved and the complexity of the decoder. Moreover, if the clock frequency of the tester is $f_{T}$ and the clock frequency of the scan chain is $f_{\text {sys }}$ (system clock frequency) then the ratio of the system clock frequency and the tester clock frequency $f_{\text {sys }}=f_{T}$ limits the minimum size of a codeword.If the test set is divided into fixed-length blocks of $b$ bits, then the serializer will hold b bits, and, thus, it takes b scanclock cycles to shift the buffer's contents into the Scan chain.

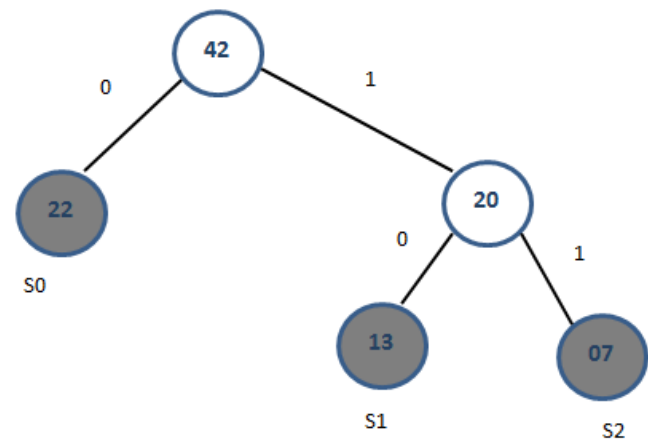

Fig.2 Huffman tree for the three highest frequency symbols

During the time that the contents of the serializer are being shifted into the scan chain, the tester is shifting bits into the decoder. When the decoder receives a complete codeword, it needs to output the corresponding block of $\mathrm{b}$ bits into the serializer. If the codeword is too short, then the serializer may not have been emptied yet which would cause a problem for the decoder. So, in order to ensure that the serializer is always empty when the decoder finishes decoding a codeword, the minimum size of a codewordL $\mathrm{L}_{\min }$ must be no smaller than the ratio of the tester and scan-clock rates times the size of each block,

$L_{\min } \geq b \frac{f_{r}}{f_{\text {sys }}}$

For example, if the block size is 8 and the scan-clock rate is twice the tester clock rate, then the minimum size of a code word is 4 . Note that if it is not possible to have the scan clock rate be faster, then the tester clock rate, then an alternative solution (as previously described) is to make the scan clock rate be twice as fast as the "effective clock rate" as seen by the decoder by simply having the tester channel feed two scan chains so that the rate that the decoder receives data from the tester is half as fast as the rate at which data can be shifted into the scan chain.Using a Huffman code would provide the maximum compression; however, it would require a complex decoder and may not satisfy the constraint on the minimum size of a codeword. Therefore, some alternative statistical code must be selected. The approach taken here involves using a selective coding approach for which a very simple decoder can be constructed. Consider the case where the test set is divided into fixedlength blocks of $\mathrm{b}$ bits. There will be $2^{b}$ Code words. The first bit of each codeword will be used to indicate whether the following bits are coded or not. If the first bit of the codeword is a 0 , then the next $b$ bits are not coded and can simply be passed through the decoder as is (hence, the complete codeword has b +1 bits).

\section{Experimental Results}

This section gives the complete details about the performance evaluation of the JPEG compression approach. Medical images of size $512 \times 512$ pixels have been taken for simulation. To obtain simulation results, Matlab software is used along with wavelet toolbox to encode Medical images of size $512 \times 512$ at a rate of 0.25 bit per pixel. The obtained results are shown below.

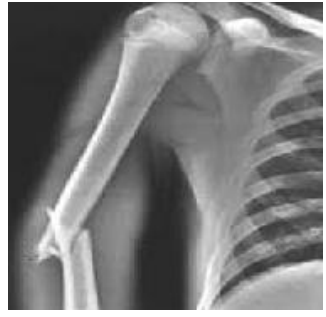

(a)

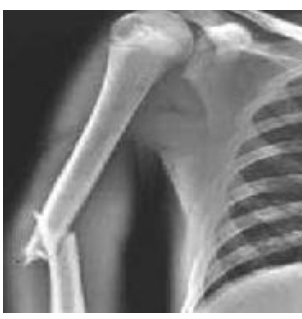

(b)

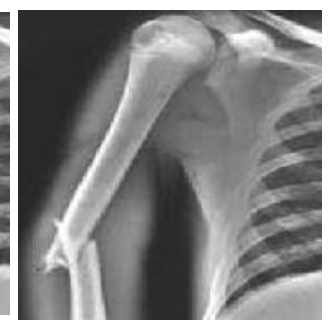

(c)

Figure3. (a) Original Image, (b) Retrieved image after JPEG coding (c) Retrieved image after hybrid coding The performance of Proposed hybrid coding is measured through performance evaluation parameters such Compression Rate (CR), Encoding Time (ET), Decoding Time (DT), Total Time(TT) for processing and Peak 
Signal to Noise Ratio (PSNR). The obtained simulation parameters for the above given test image along with the earlier JPEG coding are shown in Table.1.

Table.1 Performance metrics

\begin{tabular}{|c|c|c|}
\hline Metric & JPEG-coding & Hybrid-coding \\
\hline Compression Rate (Bpp) & 1.25 & 2.8234 \\
\hline Encoding Time Taken $(\mathrm{sec})$ & 5.125 & 6.8438 \\
\hline decoding Time Taken $(\mathrm{sec})$ & 9.0625 & 2.5469 \\
\hline Total processing Time Taken (sec) & 14.1875 & 9.3906 \\
\hline PSNR (dB) & 40.2530 & 50.2530 \\
\hline
\end{tabular}

In a similar, manner more samples are given for testing and the obtained results are shown below.

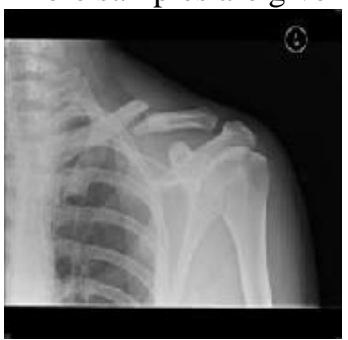

(a)

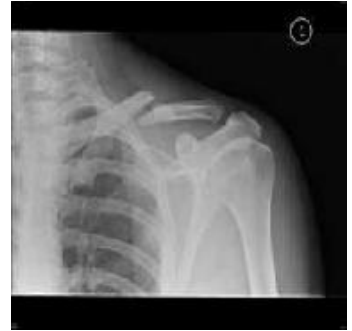

(b)

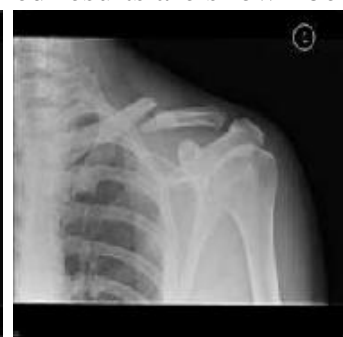

(c)

Figure.4 (a) original Image, (b) Retrieved image after JPEG coding (c) Retrieved image after Hybrid coding

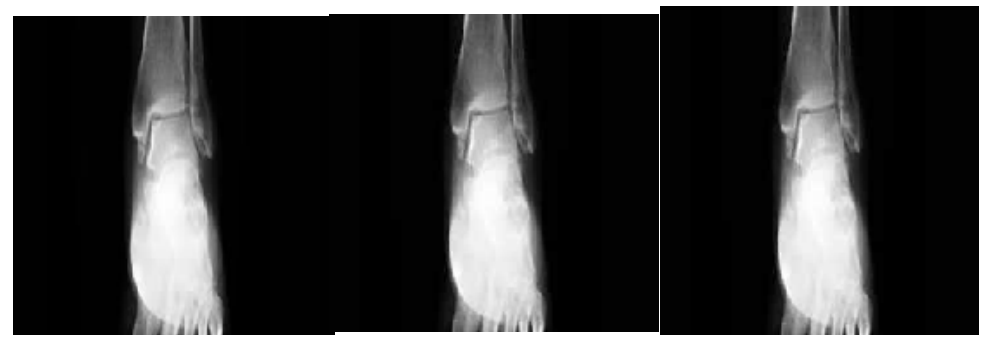

(a)

(b)

(c)

Figure.5. (a) original Image, (b) Retrieved image after JPEG coding (c) Retrieved image after Hybrid coding

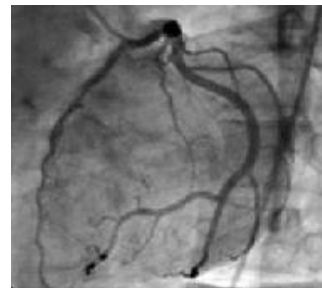

(a)

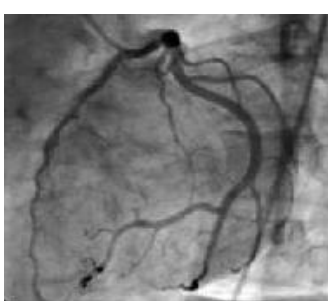

(b)

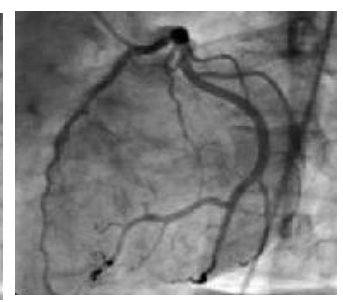

(c)

Figure.6 (a) original Image, (b) Retrieved image after JPEG coding (c) Retrieved image after Hybrid coding

Table. 2 performance metrics for various test samples

\begin{tabular}{|c|c|c|c|c|c|c|}
\hline \multirow{2}{*}{ Metric } & \multicolumn{2}{|c|}{ Sample-1 } & \multicolumn{2}{c|}{ Sample-2 } & \multicolumn{2}{c|}{ Sample-3 } \\
\cline { 2 - 6 } & JPEG & $\begin{array}{c}\text { Hybrid } \\
\text { coding }\end{array}$ & JPEG & $\begin{array}{c}\text { Hybrid } \\
\text { coding }\end{array}$ & JPEG & $\begin{array}{c}\text { Hybrid } \\
\text { coding }\end{array}$ \\
\hline Compression Rate & 2.0156 & 3.4937 & 1.2031 & 2.7625 & 1.6719 & 3.25 \\
\hline Encoding Time & 5.2188 & 6.8125 & 4.6406 & 7.4375 & 5.25 & 6.9688 \\
\hline decoding Time & 9.4063 & 2.0781 & 7.2188 & 2.0469 & 9.7656 & 2.7656 \\
\hline Total processing Time & 14.625 & 8.8906 & 11.8594 & 9.4844 & 15.0153 & 9.7344 \\
\hline PSNR (dB) & 39.8869 & 49.8869 & 42.5901 & 52.5901 & 39.5556 & 49.5556 \\
\hline
\end{tabular}

To evaluate the quality assessment for the processed data the quality metric of peak signal to noise ratio (PSNR), mean square error (MSE) and spatial similarity index measure (SSIM) is used, tested over different noise density and coding factor. PSNR is most commonly used as a measure of quality of image test sample .Peak signal-to-noise ratio is defined as a ratio between the maximum possible power of a signal and the power of corrupting noise that affects the fidelity of its representation. PSNR is usually expressed in terms of the logarithmic decibel scale. 
$\operatorname{PSNR}(d B)=10 \log _{10}\left(\frac{I_{\text {peak }}^{2}}{M S E}\right)(2)$

Where $I_{\text {peak }}$ is the peak values of the input video. Peak signal to noise ratio is used to evaluate the quality of data after retrieval process. The Mean squared error (MSE) of an estimator is one of the way to quantify the amount by which an estimator differs from the true value of the quantity being estimated. As a loss function, MSE is called squared error loss. MSE measures the average of the square of the error. The error is the amount by which the estimator differs from the quantity to be estimated defined by,

$M S E=\frac{1}{M X N} \sum(f-\hat{f})^{2}(3)$

Where $\mathrm{f}$ is the ground truth video and $\hat{f}$ is the interpolated video after extraction. The SSIM, similarity factor establishes the similarity of pixel intensities between the ground truth test sample and the decoded data, given as,

$\operatorname{SSIM}=\frac{\sum_{i} \sum_{i} f(i, j) \otimes f_{w}(i, j)}{\sum_{i} \sum_{i}(f(i, j))^{2}}(4)$

The similarity factor is approximately nearer to 1 for better imperceptible data quality. The measured observations are presented in figure below. The obtained simulation parameters for the above given test image along with the earlier JPEG coding are shown in the table.2.

Table.3 Performance metrics

\begin{tabular}{|c|c|c|c|}
\hline Metric & JPEG & HYBRID & Sel-Hybrid \\
\hline Compression Rate (Bpp) & 1.25 & 2.8234 & 3.475 \\
\hline Encoding Time Taken (sec) & 5.125 & 6.8438 & 5.125 \\
\hline decoding Time Taken (sec) & 9.0625 & 2.5469 & 2.3281 \\
\hline Total processing Time (sec) & 14.1875 & 9.3906 & 7.4531 \\
\hline PSNR (dB) & 40.2530 & 50.2530 & 50.6670 \\
\hline
\end{tabular}

Similarly, some more samples are given for testing and the obtained results are shown below.

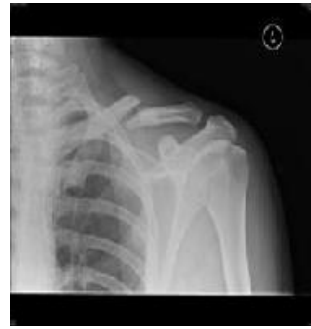

(a)

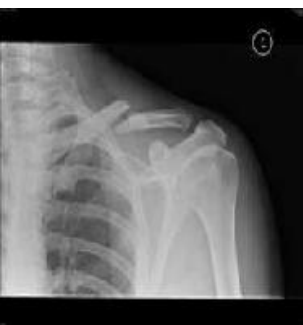

(b)

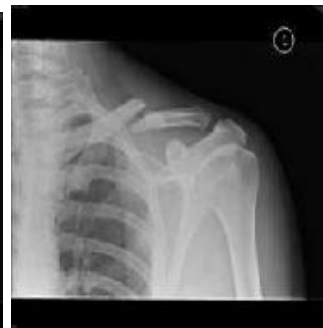

(c)

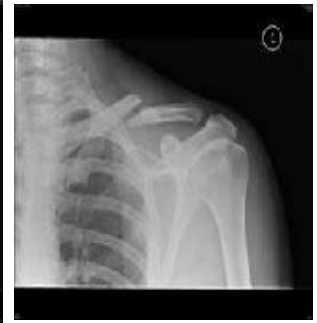

(d)

Figure.7 (a) original Image, (b) Retrieved image after JPEG coding (c) Retrieved image after HYBRID coding (d) Retrieved image after Sel-Hybrid coding

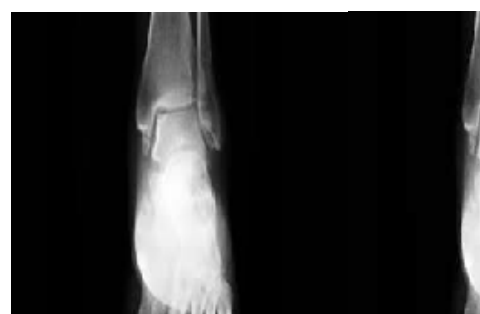

(a)

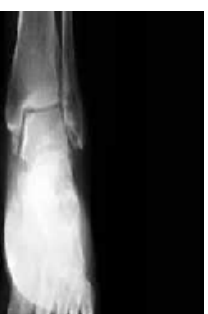

(b)

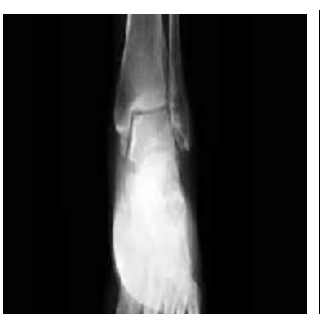

(c) (d)

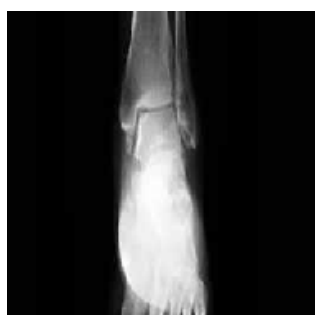

Figure.8 (a) original Image, (b) Retrieved image after JPEG coding (c) Retrieved image after HYBRID coding (d) Retrieved image after Sel-Hybrid coding

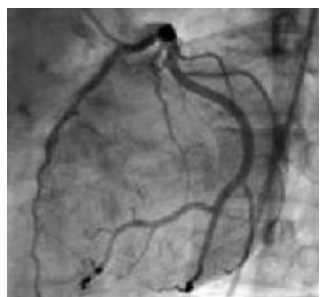

(a)

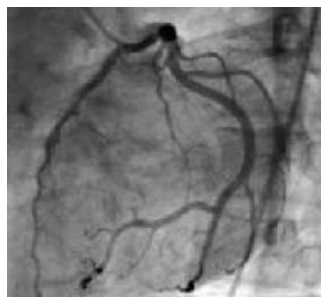

(b)

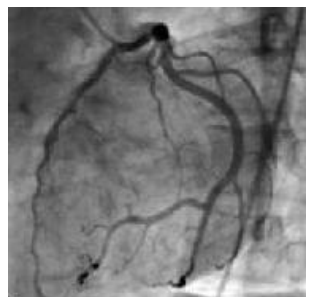

(c)

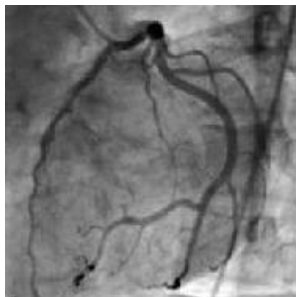

(d)

Figure.9 (a) original Image, (b) Retrieved image after JPEG coding (c) Retrieved image after HYBRID coding (d) Retrieved image after Sel-Hybrid coding 
Table.4 performance metrics for various test samples

\begin{tabular}{|c|c|c|c|c|c|c|c|c|c|}
\hline \multirow{2}{*}{ Metric } & \multicolumn{3}{|c|}{ S1 } & \multicolumn{3}{c|}{ S2 } & \multicolumn{3}{c|}{ S3 } \\
\cline { 2 - 10 } & JPEG & HYBRID & Sel-Hybrid & JPEG & HYBRID & $\begin{array}{c}\text { Sel- } \\
\text { Hybrid }\end{array}$ & JPEG & HYBRID & $\begin{array}{c}\text { Sel- } \\
\text { Hybrid }\end{array}$ \\
\hline CR & 2.015 & 3.493 & 4.3 & 1.203 & 2.762 & 4.075 & 1.671 & 3.253 & 4.00 \\
\hline ET & 5.218 & 6.812 & 4.468 & 4.640 & 7.437 & 4.781 & 5.255 & 6.968 & 4.531 \\
\hline DT & 9.406 & 2.078 & 2.187 & 7.218 & 2.046 & 2.187 & 9.765 & 2.765 & 2.25 \\
\hline TT & 14.62 & 8.890 & 6.653 & 11.85 & 9.484 & 6.968 & 15.01 & 9.734 & 6.781 \\
\hline PSNR & 39.88 & 49.88 & 50.309 & 42.59 & 52.590 & 50.62 & 39.55 & 49.555 & 49.96 \\
\hline
\end{tabular}

The obtained simulation parameters for the above given test image along with the earlier JPEG coding are shown in the table.5.

Table.5 Performance metrics

\begin{tabular}{|c|c|c|c|c|}
\hline Metric & JPEG & HYBRID & Sel-Hybrid & JPEG-Sel-Hybrid \\
\hline Compression Rate & 1.25 & 2.8234 & 3.475 & 4.126 \\
\hline Encoding Time Taken & 5.125 & 6.8438 & 5.125 & 10.625 \\
\hline decoding Time Taken & 9.0625 & 2.5469 & 2.3281 & 2.187 \\
\hline Total processing Time Taken & 14.1875 & 9.3906 & 7.4531 & 12.8125 \\
\hline PSNR (dB) & 40.2530 & 50.2530 & 50.6670 & 53.263 \\
\hline
\end{tabular}

Similarly, some more samples are given for testing and the obtained results are shown below.

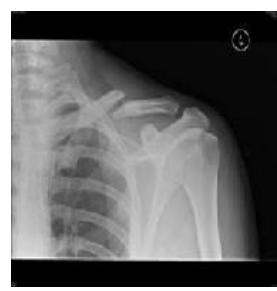

(a)

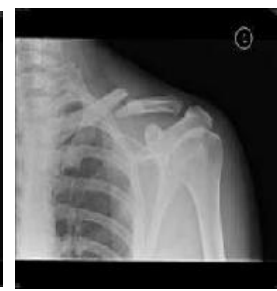

(b)

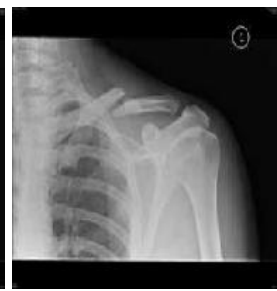

(c)

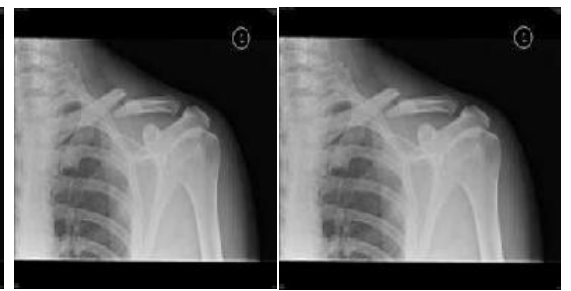

(d) (e)

Figure.10 (a) original Image, (b) Retrieved image after JPEG coding (c) Retrieved image after HYBRID coding (d) Retrieved image after Sel-Hybrid coding (e) Retrieved image after JPEG-Sel-Hybrid coding

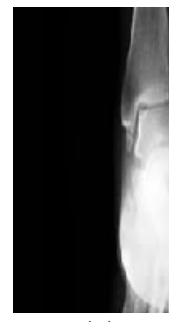

(a)

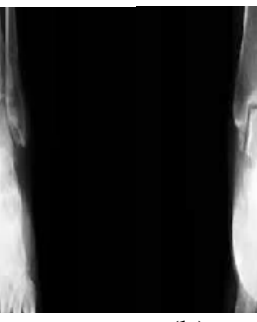

(b)

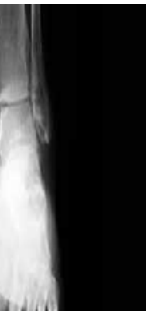

(c)

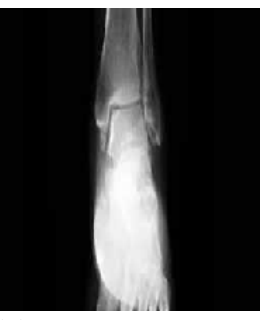

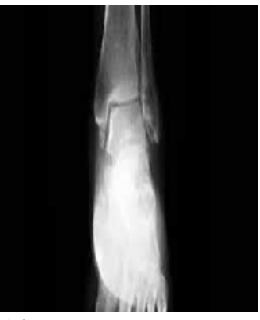

(d)

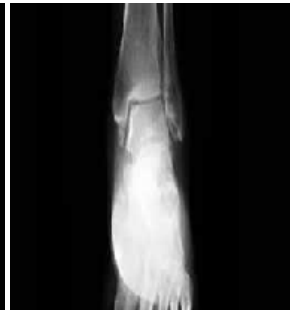

(e)

Figure.11 (a) original Image, (b) Retrieved image after JPEG coding (c) Retrieved image after HYBRID coding (d) Retrieved image after Sel-Hybrid coding (e) Retrieved image after JPEG-Sel-Hybrid coding

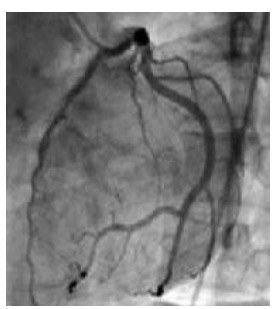

(a)

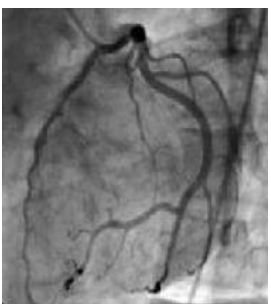

(b)

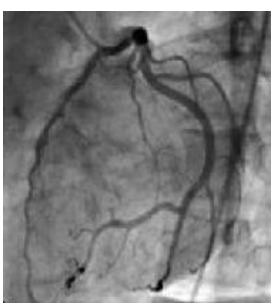

(c)

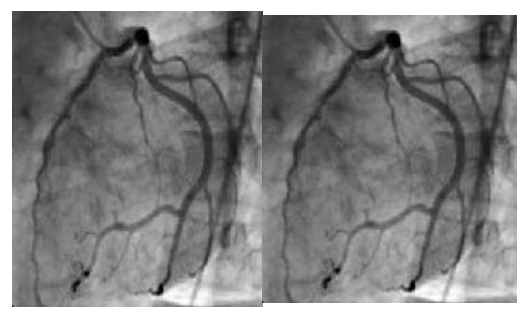

(d) (e)

Figure.12 (a) original Image, (b) Retrieved image after JPEG coding (c) Retrieved image after HYBRID coding (d) Retrieved image after Sel-Hybrid coding (e) Retrieved image after JPEG-Sel-Hybrid coding 
Table.6 performance metrics for various test samples

\begin{tabular}{|c|c|c|c|c|c|c|c|c|}
\hline \multirow{2}{*}{ Metric } & \multicolumn{3}{|c|}{ S1 } & \multicolumn{3}{c|}{ S2 } \\
\cline { 2 - 9 } & JPEG & Hybrid & Selective & Sel-Hybrid & JPEG & Hybrid & Selective & Sel-Hybrid \\
\hline CR & 2.015 & 3.493 & 4.3 & 5.10 & 1.203 & 2.762 & 4.075 & 4.03 \\
\hline ET & 5.218 & 6.812 & 4.468 & 7.93 & 4.640 & 7.437 & 4.781 & 8.03 \\
\hline DT & 9.406 & 2.078 & 2.187 & 2.56 & 7.218 & 2.046 & 2.187 & 1.93 \\
\hline TT & 14.62 & 8.890 & 6.653 & 10.0 & 11.85 & 9.484 & 6.968 & 9.96 \\
\hline PSNR & 39.88 & 49.88 & 50.30 & 52.8 & 42.59 & 52.59 & 50.62 & 55.6 \\
\hline
\end{tabular}

\section{Conclusion}

The JPEG Sel-Hybrid model gives a higher performance in image compression as compared to the conventional model of JPEG based coding. It is also observed that the isolated method of Neural network based image compression and Sel-Hybrid logic based image compression results in lower accuracy as in comparison to the integrated model of neural network and Sel-Hybrid logic. The Sel-Hybrid rules and the learning capability of the neural network, leads to a proper selection of pixel for coding which result in higher compression ratio with low MSE and higher PSNR value.

\section{References}

[1] Gregory K Wallace , “The JPEG Still Picture Compression Standard”, IEEE Transactions on Consumer Electronics, vol. 38, Issue 1, pp. xvii - xxxiv, 1992.

[2] Bryan E. Usevitch, "A Tutorial on "Modern Lossy Wavelet Image Compression: Foundations of JPEG 2000", IEEE signal processing magzine, 1053-5888,sep-2001.

[3] J. Jyotheswar, SudiptaMahapatra, "Efficient FPGA implementation of DWT and modified SPIHT for lossless image compression", Journal of Systems Architecture, Vol.53, pp.369-378, 2007

[4] David taubmann "high performance scalable image compression with EBCOT" IEEE transactions on image processing, vol. 9, no. 7, july 2000

[5] Ms. P.Srikala, Mr .Shaik Umar, "Neural Network Based Image Compression with Lifting Scheme and RLC” IJRET, SEP 2012.

[6] [6] Ryuji Matsuoka*, MitsuoSone, KiyonariFukue, Kohei Cho, HaruhisaShimoda., "Quantitative Analysis of Image Quality Of Lossy Compression Images".

[7] S. Grace Chang, Gregory S. Yovanof "A Simple Block-Based Lossless Image Compression Scheme”IEEE 1997

[8] Yao-Tien Chen, Din-Chang Tseng, and Pao-Chi Chang "Wavelet-based Medical Image Compression with Adaptive Prediction" IEEE 2005.

[9] Wade Spires, “Lossless Image Compression via the Lifting Scheme”, November 2005

[10] David taubmann "high performance scalable image compression with EBCOT" IEEE transactions on image processing, vol. 9, no. 7, july 2000

[11] Ms. P.Srikala, Mr .Shaik Umar, "Neural Network Based Image Compression With Lifting Scheme and RLC” IJRET, SEP 2012.

[12] ChigozieObiegbu, "Image Compression Using Cascaded Neural Networks", University of New Orleans 2003.

[13] UdoSeiffert, “ANNIE_-Artificial NeuralNetwork-based Image Encoder”, Neurocomputing, Volume 125, 11 February 2014, Pages 229-235

[14] Yao-Tien Chen, Din-Chang Tseng, and Pao-Chi Chang "Wavelet-based Medical Image Compression with Adaptive Prediction" IEEE 2005.

[15] Wade Spires, "Lossless Image Compression Via the Lifting Scheme", November 2005.

[16] AbirJaafarHussain,Dhiya Al-Jumeily, NaeemRadi, Paulo Lisboa' "Hybrid Neural Network Predictive-Wavelet Image Compression System”, Neurocomputing, Elsevier, Volume 151, Part 3, Pages 975-984, 2015.

[17] W. K. Yeo, David F. W. Yap, T.H. Oh, D.P. Andito, S. L. Kok, Y. H. Ho, M. K. Suaidi, "Grayscale Medical Image Compression Using Feed forward Neural Networks", International Conference On Computer Applications And Industrial Electronics, Pp 633$638,2011$.

[18] S. Anna Durai, and E. Anna Saro," Image Compression with Back-Propagation Neural Network using Cumulative Distribution Function," International Journal of Engineering and Applied Sciences 2007.

[19] Saleh Ali Alshehri, "Neuralnetwork technique for imagecompression “, IET Image Processing,Volume: 10, Issue: 3Pages: 222 $226,2016$.

[20] S.Jagdeesh, "Multi-Spectral Band Selective Coding for Medical Image Compression", Global Journal of Computer Science and Technology, Volume 15 Issue 3 Version 1.0 Year 2015. 